\title{
The Distance Between Us
}

\author{
Mike Curtis*
}

As the Internet morphs into cyberspace, the relationships forged online can become as significant as those arising by more traditional means. Cyberspace is the environment created from global connected technology; a virtual world where people can meet, engage in discussions, conduct business, play and create an almost complete analogue of the physical world. Many are questioning the concept of the Nation State as the primary political entity and some online communities are beginning to take on the trappings of statehood, holding elections and raising taxes but without any clear understanding of what defines their society. The selection of appropriate communities to join, and other individuals with whom to communicate, becomes more difficult. The amount of choice when it comes to joining online communities can be bewildering; relying on word of mouth can severely limit choice, and the only help comes from commercial organisations using algorithms and Artificial Intelligences whose workings are unknown and whose recommendations are based on maximising profit for the company rather than the ultimate benefit of the user. This article proposes mechanisms that are open, can be used by individuals and communities and, where recommendation systems are used to help, their workings are transparent.

\section{Introduction}

Human beings are social animals and have always lived in groups. The earliest groupings, those of family and tribe where the connections between members were based on emotion and shared enterprise, were extended as permanent settlements arose, leading to additional geographical connections. For most people there was little or no choice as to the groups to which they belonged; movement between groups was possible but often difficult. As the technologies for collectively concentrating energy and food improved enabling long-distance travel and communi-

\section{DOI: $10.21552 /$ delphi/2019/1/4}

* Mike Curtis MA PGCE C.Eng MBCS CITP is a retired IT consultant and sometime Computer Science lecturer with a lifelong interest in the way technological change affects social and political structures. He can be contacted at $<$ mike@mullsoft.co.uk>

1 T Schelling, 'Models Of Segregation' (1969) 59 American Economic Review

2 W Clark and M Fossett, 'Understanding The Social Context Of The Schelling Segregation Model' (2008) 105 Proceedings of the National Academy of Sciences

3 'The Politics Of Place And Polarization - Electoral Geographies' (Electoralgeographies.web.unc.edu, 2018) <http:// electoralgeographies.web.unc.edu/how-political-segregation-has -changed-american-electoral-geography> accessed 11 October 2018 cation, other, wider groupings became possible; scholars could correspond, religions could unify and extend their influence, and political entities, city states and then nation states emerged.

Despite the improvements in travel and communication, the major factor in determining what constituted a community remained geography. In 1969 Thomas Schelling's Models of Segregation demonstrated how people who felt a close connection to each other tended to move so that they lived in close geographical proximity. ${ }^{1}$ People always seem to prefer to live in a community of people that are close to them in other non-geographical ways. This result remains the basis for much research and has been used to model the growth in particular of racial segregation and political polarization. ${ }^{2,3}$ These communities within which we live serve not only to provide the social needs and economic needs for businesses, but they also provide the basis for political organization whereby, ideally at least, each community elects one or more representatives to the overarching political entity, the state. The natural desire of most people is to belong to communities of people that are 'just like us.'

In 1954 Martin Heidegger in his lecture 'The Question Concerning Technology' noted that because of technology, 'all distances in time and space are 
shrinking' and 'yet the hasty setting aside of all distances brings no nearness; for nearness does not consist in a small amount of distance. ${ }^{4}$ He warned of the potential dangers in the way that technology becomes interwoven in the fabric of society and hence limits experience, though he dismissed the notion that technology should be rejected, rather that its dangers should be understood.

The social use of technology, in particular using Computer Mediated Communication, ${ }^{5}$ is now endemic throughout our society and the consequences are beginning to be understood. There are dangers, as shown in the work of Kross et al, who have shown how the use of Facebook by young adults can lead to a measurable decrease in subjective well-being. ${ }^{6}$ However, Ridings and Gefen, in their study, showed that users sought and found friendship and social support through their online relationships. ${ }^{7}$

Many real world communities are formed and grow as individuals gradually discover common ground which they wish to explore together; individuals may communicate regularly in pairs or small subgroups with occasional fuller meetings to explore specific ideas. Individuals are usually free to participate in specific activities or not, with the proviso that continued non-participation may call membership into question. Online communities tend to start with a specific topic or attitude and individuals with a relevant interest join up, often with little or no knowledge of the other members, and communication tends to be fairly continuous and rarely involve everybody. The act of communication, and the search for suitable communities or suitable individuals is usually by means of commercial organisations whose primary concern is to promote use of their platform and thus increase the consequent advertising revenue. This can result in a large space of possible communities which is difficult to navigate and may lead to less successful outcomes for the individual, though no doubt commercial success for the platform owners.

This articles sets out some of the characteristics of virtual communities, and how we might be able to define and calculate a measure of the distance between individuals and communities and thus help to classify communities, determine which ones to join so as to enrich our lives and to enable such communities to function in a similar way to those in the real world. Such a measure should take into account as many as possible of the different ways that people relate to each other, and their reasons for doing so. It must also provide a meaningful result that can be readily understood by human beings, and be calculated using an algorithm that can be manually applied and is not hidden within a complex computer program, although for convenience computers will normally be used to perform both calculation and presentation of results. The results should then be used as a guide to making choices which will always retain a subjective element. The eventual aim is to provide a standard terminology and mechanism for describing online relationships which can be embedded with a suitable algorithm within a system that can be used as an aid in finding candidates and assessing suitability for forming online relationships.

\section{Communities}

The Internet now provides the opportunity to define new virtual communities for which the geographical connection becomes much less relevant. The feelings of closeness and mutual support which are needed to satisfy the social requirements of our psychological nature become possible, at least in part, without the need for physical proximity. I may still have common interests with my geographical neighbours in maintaining the local infrastructure, and we may be on friendly terms but, by choice, when I want to socialize with others, I can and most likely do choose from many and wide circles of friends scattered all around the world. For many of the subjects in which I am interested, I have nothing in common with my physical neighbours but feel most close to people that I may well have never physically met and quite possibly never will. Our environment and upbringing will also naturally predispose us, consciously or unconsciously, to belong to certain groups. ${ }^{8}$ For many

4 Martin Heidegger and William Lovitt, The Question Concerning Technology, and Other Essays (HarperCollins Publishers, 2013)

5 Computer Mediated Communication is any communication between human beings using two or more electronic devices

6 E Kross, P Verduyn, E Demiralp, J Park, DS Lee, N Lin et al, 'Facebook Use Predicts Declines in Subjective Well-Being in Young Adults' (2013) PLoS ONE 8, 8

7 Catherine M Ridings and David Gefen, 'Virtual Community Attraction: Why People Hang Out Online' (2004) 10 Journal of Computer-Mediated Communication 1

8 A Fiske et al, 'The Cultural Matrix of Social Psychology' in D Gilbert, S Fiske and G Lindzey (eds), The Handbook of Social Psychology (McGraw-Hill, 1998) 
people and many activities, there can be no substitute for face to face physical connections, but it seems likely that this will decrease for generations who have grown up with and in cyberspace.

First we must make clear what we mean by community. It is quite common now to refer to any group discussing online one or more topics of mutual interest as a community. I am adopting a more strict definition, which is a permanent or semi-permanent group of individuals who maintain reasonably continuous contact with each other and which constitutes a significant factor in their life. Examples are Second Life, ${ }^{9}$ which has tried to mimic real world communities in a virtual environment; Asgardia, ${ }^{10}$ which assumes that the type of society that it is trying to create will eventually be realised in a space habitat and The Good Country, ${ }^{11}$ which seeks to bring together people dissatisfied with current global politics, specifically the antagonism between nations and the disregard for the environment. Such communities must have a clear definition of membership, some sort of governing body (which may be all of its members), a set of laws governing the behaviour of members, sanctions for those who break the laws and mechanisms to resolve disputes. They may also need a means of raising revenue by some form of taxation.

As with most matters relating to the Internet, the number of such communities is likely to increase rapidly and, while it may be perfectly possible to belong to more than one, as with maintaining homes in two geographical communities, the amount of commitment to each is bound to decrease while the expense increases. It therefore becomes increasingly important that we have some ways of analysing and classifying these collections of people, first to assess whether they are a genuine community with

9 R Metz, 'Second Life Is Back for a Third Life, This Time in Virtual Reality' (MIT Technology Review, 27 January 2017) <https://www .technologyreview.com/s/603422/second-life-is-back-for-a-third -life-this-time-in-virtual-reality> accessed 11 October 2018

10 'Asgardia - The Space Nation' (Asgardia.space, 2018) <https:// asgardia.space/en/> accessed 18 October 2018

11 'The Good Country' (The Good Country, 2018) <https:// goodcountry.org/> accessed 18 October 2018

12 A graph in this sense is a collection of points or vertices connected by lines or edges. The vertices and/or edges may be labelled; often the label is numeric in which case it may be termed a weight. Most commonly a weighted graph would be one where the edges had numeric labels. Edges may or may not have a specified direction. common goals and a clearly defined structure and then to determine whether our individual interest and beliefs are such as to make community membership desirable. This is especially important if or when online communities take on some political role as the influence of traditional nation states wanes. Many people will already belong to a number of putative communities using current communication mechanisms such as email lists or Facebook groups, mostly we will have found these groups by word of mouth, simple keyword searches, relevance to existing interests or as a result of a commercial recommendation system. The first three of these methods are reasonably reliable, but limited, so that it is easy to completely miss groups that would be more relevant and interesting than the ones we have joined. Commercial systems are becoming more sophisticated but their motives and means are both suspect; the algorithms are usually commercial secrets and their purpose is to increase profits for their owners. This does not necessarily mesh very well with our actual requirements. Inevitably, given the size of the Internet, we need help navigating and assessing the very large number of candidates we may find. Given the chance to make a wider search, we may also need help expressing our requirements in such a way that others can find us. Apps, algorithms and artificial intelligence all have their uses, however it seems essential that the human element is retained and that we maintain control over the process. Our existing communications, purchases, comments, likes and dislikes may be used to prepare a profile, but we would like to know how that was done and given the opportunity to amend it. If some other individual or community is recommended as of potential interest then that information should be presented along with their profile and reasons why the match was chosen as suitable.

\section{Social Networks}

Social Network Analysis is the investigation of individuals and the relationships between them as nodes and links in a network. A common mechanism for representing such social network is by means of a weighted graph. ${ }^{12}$ The individuals are represented by the vertices of the graph and their communication by edges. Edge weights are a measure of the degree of contact between the two connected individuals which 


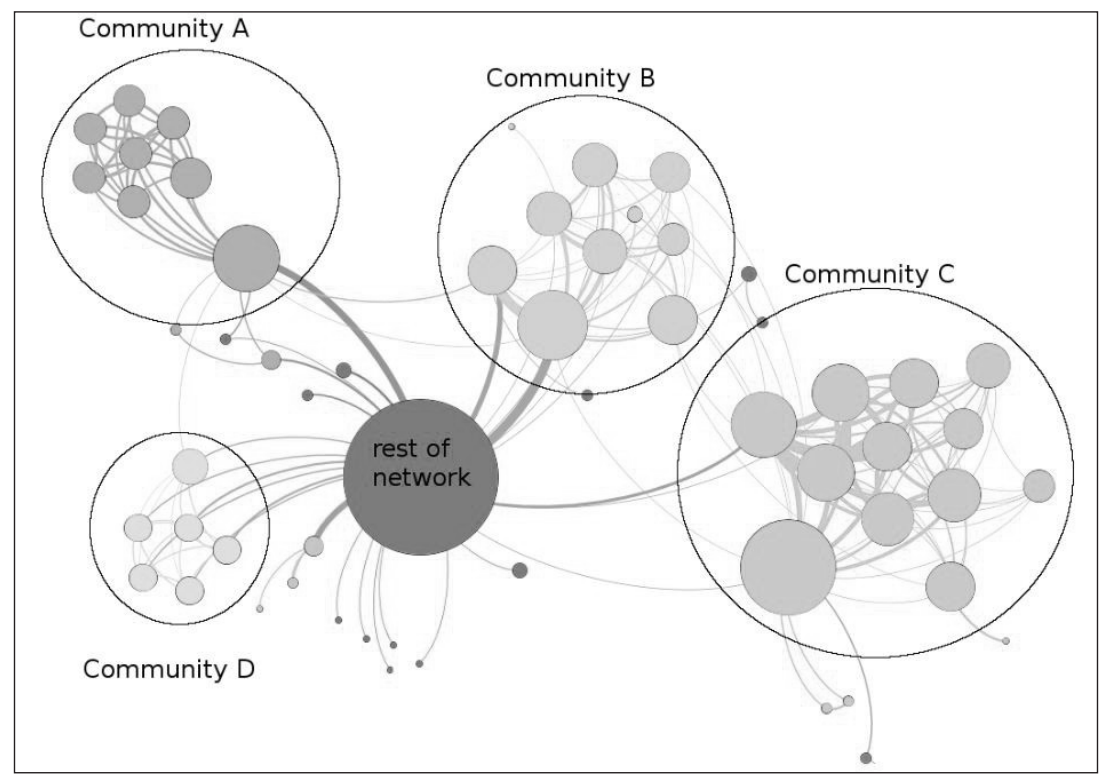

Figure 1: A social network graph showing clustering

Source: Author's elaboration

may simply be the volume of traffic, or may involve analysis of the actual content of the messages to evaluate the actual attraction between them. ${ }^{13}$ Various algorithms exist, or are being researched, to detect clusters, which would be a relatively small subset of vertices between which the degree of communication as measured by the weights of the corresponding edges remains large and fairly static. ${ }^{14}$ These clusters are then called 'communities.' This work is important for commercial reasons; it improves the detection of individual preferences and opportunities for targeted advertising. This way of looking at online communities from the outside, for commercial exploitation or other reason is useful, but not particularly germane to our current discussion, where we are assuming that the individuals within a community have self-identified as such, and published their defining characteristics, and we wish to measure ourselves against those characteristics to assess our membership or prospective membership of the community. This has to be in part a subjective assessment, though one which could be simplified or even codified. The techniques do however remain useful in order to measure the degree of coherence within the community and any possible drift in purpose over time.

Figure 1 Is an example of a graph illustrating the connections between a set of individuals where the number of links between nodes has been used to determine a number of clusters, which indicate the existence of putative communities. In this example the thickness of an edge is used to show a measure of the connection between the nodes and the size of a vertex to illustrate the volume of traffic involving that node.

The first problem to tackle is the use of simple numbers as our weights to measure the degree of attraction between two vertices or individuals. The connections between human beings are too complex to be mapped onto a single number. Two people may be connected to the point of maintaining communication for a variety of reasons, each of which may be of more or less importance in any particular context and any single reason, or any combination of reasons, may be sufficient to stimulate first and subsequent contact. There are, of course, an almost infinite variety of ways in which human beings can relate to each other, which must be reduced to a man-

13 A Kanavos et al, 'Emotional Community Detection in Social Networks' (2017) 65 Computers \& Electrical Engineering 449

14 R Liu et al, 'Weighted Graph Clustering for Community Detection of Large Social Networks' (2014) 31 Procedia Computer Science 85 
ageable number. There are certain qualities possessed by individuals which are largely objective and rarely, if ever, change, or change slowly. Examples are age, race and gender. These have been omitted from our consideration and left, where relevant, to the subjective interpretation of results. The way these influence interpretation will vary across cultures, and in many cultures anything that could be considered as discrimination based on these qualities is illegal. The remaining facets that could describe aspects of human social intercourse have to be pared down to a set that is mutually independent, large enough to retain all relevant information, yet small enough to be readily appreciated by the user and amenable to mathematical manipulation. Some limits for human judgement and memory were set out by G.A.Miller in 1956 as seven, plus or minus two. ${ }^{15}$ While a tuple, or ordered collection of numbers, can be of any length, arithmetic is particularly convenient and well studied for two, four or eight elements.

I have therefore chosen eight facets which can be assigned numeric values to represent both the relative importance to the individual of the facet and the degree of compatibility between two individuals as well as the average or combined indicator of the nature of the community. Other choices are possible, the calculations and interpretation of the results remaining essentially the same. The eight values could comprise an octonion, ${ }^{16}$ for which there is already a distinct mathematics and which are becoming increasingly important in other areas, such as String Theory. ${ }^{17} 18$

They may also be considered as a vector, or point in eight dimensional space. Two relatively unusual features of octonion arithmetic do however reflect correctly the properties of this particular domain. Multiplication of octonions is not commutative, that

15 G A Miller, 'The magical number seven, plus or minus two: Some limits on our capacity for processing information' (1956) 63 Psychological Review 2, 81-97

16 An octonion is a hypercomplex number with eight elements; it is in effect an eight element vector or a point in eight dimensional space. They form one of the only four division algebras along with real numbers ( 1 element), complex numbers ( 2 elements) and quaternions (4 elements).

17 JC Baez, 'The Octonions' (2002) 39 Bulletin of the AMS 145

18 Natalie Wolchover, 'The Octonion Math That Could Underpin Physics' (Quanta Magazine, 2018) <https://www.quantamagazine .org/the-octonion-math-that-could-underpin-physics-20180720/> accessed October 112018 is the order of multiplication matters, $\mathbf{a} \cdot \mathbf{b} \neq \mathbf{b} . \mathbf{a}$, where $\mathrm{a}$ and $\mathrm{b}$ are octonions representing individuals or communities and '.' represents the octonion equivalent of multiplication which, in our calculus, will be to form a representation of the relationship between $a$ and $b$, in effect the community formed from a and b. Communications or relationships between a and $\mathbf{b}$ are clearly not the same as those between $\mathbf{b}$ and $\mathbf{a}$. For example if the connection represented is is attracted to' then Anne being attracted to Bill is clearly not the same thing as Bill being attracted to Anne. Much human activity such as storytelling and learning revolves around the development of contextual non-commutative sequences. They are also not associative, which means that when three are multiplied, the answer is different depending on which pair is multiplied first, (a.b).c $\neq$ a.(b.c). For example the pair of Anne and Bill, connected because Anne is attracted to Bill, have a mutual friend Clive; this is not the same as Anne being attracted to the pair Bill and Clive who are connected because they have a mutual friend. These examples are not meant to imply that simple multiplication is used to form community relationships but that any formula that is used when octonions are used as edge weights, which are the values associated with the links between graph nodes, will therefore also be probably non-commutative and non-associative and this does reflect the situation for communications and relationships between individuals and communities. It also correctly reflects the way that such connections are bidirectional, but with different interpretations in the two directions.

\section{Eight Facets}

These are the eight facets which we will use to define the nature of communities and personal preferences. These affect the way communities form and an individual's choice of appropriate community. For each its importance can vary from completely irrelevant to absolutely vital. By assigning to each a numerical value to indicate importance and a short textual description we obtain a profile of the individual or community that can be used to determine the strength of a connection that they may form. An algorithm can take a comparison of text values which can be either subjective or by textual analysis to provide weights that can be applied to the numerical calcula- 
tion using the two importance factors to provide a numerical measure for that facet. By studying the results of the calculation, two individuals and communities gain useful information of the closeness of the match between them which assists them in the decision whether to join a community or initiate an online relationship.

\section{Geographic}

For most people a geographic base is essential, and this implies an immediate close connection with those living in the same area. How far this area extends is a matter for debate. Clearly there are important mutual concerns with the local infrastructure; the supply of services, participating in some leisure activities, the availability of basic goods and, within limits, opportunities for employment. As examples such as the Schengen Area within the European Union have shown, ${ }^{19}$ it makes virtually no difference if the next town or village is in a different jurisdiction, has different religious practices, holidays, architectural styles or history. In a world of plastic money and automatic translation, even different currencies and languages become irrelevant. There are also larger scale differences such as the divides between global north and south and between east and west that may be relevant, and which may influence the way that other factors, such as cultural ones, may be considered.

\section{Emotional}

Most people have family and friends. The family is one community into which one is born and, for most people, remain; it is outside the realms of this discussion since there is no element of choice. Most of us choose our friends and, while there may be other factors that bring us together, friendship is distinguished by an emotional bond. In general we like our friends and desire to remain in contact even when there is very little else that we have in common. Often contact diminishes over time and, for many of us, friendship can survive with little or no contact. Conversely we do not need to feel friendship with those with whom we spend most time, for others it may be an essential element of a community.

\section{Philosophical}

This reflects the degree to which an individual or community displays an interest in the way that the world works both physically, incorporating science, logic and mathematics, but also philosophical concepts such as the relationship between mind and body and the study of truth and meaning. At its extreme, it may be as fine grained as one particular philosophical school of thought but, more generally, it is the importance of such matters rather than specific ideas.

\section{Political}

Many people relish political argument and for some they will not countenance any contact with their political enemies. Politics, along with race, are the major reasons for the sort of segregation explored by Schelling.

\section{Tribal}

This, which includes race and nationality, is, like politics, a major reason for geographical segregation. Partly this is because members of one of the many diasporas will naturally, when in a different society from the one they are used to, seek others with shared experiences and some common history. It can also be because nation states have a long history of warfare, conquest and colonial exploitation that still colours the relations between people long after it has ended; in far too many cases it is still happening. It should not, of course, be used in order to apply some form of illegal or unethical discrimination. It remains to be seen for how long the legacies of mistrust remain in virtual societies.

\section{Spiritual}

For many, belief in some spiritual dimension is important, which may extend to belief in a creator God,

19 The Schengen Area is an area comprising 26 European states that have officially abolished passport and all other types of border control at their mutual borders. 
or even to one particular religion or sect. Some consider themselves spiritual beings, but deny the existence of a God, others reject all but the physical world.

\section{Cultural}

This can be the relative importance of creativity and leisure activities in an individual's life. It can be as detailed as specific taste in music, painting or drama, or being engaged in shared activity. It can be more general as in frequent attendance at concerts and visits to art galleries.

\section{Commercial}

'Money makes the world go round' so a lot of life experiences and outlook are determined by business. This can be as specific as being employed in the same industry or by the same firm, or more general as a common interest in the operation of markets and the accompanying paraphernalia of commerce.

\section{Profiles, Descriptions and Matches}

Having chosen a suitable collection of facets, we now require a notation and calculus to formulate an algorithm which will take the numerical and textual values that we assign for the facets and provide a meaningful result. The basic items in our calculus of facets will be pairs of vectors of eight elements, one numeric, representing the values assigned to the relative importance of each of the facets above. The other is textual consisting of brief explanations for each facet.

For example $A=\left\{\left[\mathbf{a}_{o}, \mathbf{a}_{1}, \mathbf{a}_{2}, \mathbf{a}_{3}, \mathbf{a}_{4}, \mathbf{a}_{5}, \mathbf{a}_{6}, \mathbf{a}_{7}\right]\right.$, $\left.\left[A_{\circ}, A_{1}, A_{2}, A_{3}, A_{4}, A_{5}, A_{6}, A_{7}\right]\right\}$.

20 The length of a vector is given, using an extension of Pythagoras' Theorem, by the square root of the sum of the squares of all its elements. Normalisation involves dividing each element of a vector by the vector's length; the result retains the relative relationship between elements but guarantees unit length making comparison between two vectors easier. It is a similar process to reducing a fraction to its lowest terms.

21 A simple one could be, for each facet, to replace any match which was less than the threshold by zero, average the source and object values, multiply by the match and subtract the result from 1 .
Since we are only concerned with relative values, we can simplify any mathematics by assuming that these are normalised with length $1 .^{20}$

The textual elements may be provided subjectively by the community or individual to which they refer; they may also be provided by analysis of existing communications and activities in a similar way to what is already done to provide targeted advertising and recommendations.

Such a pair is referred to as a profile. So, for example a profile of

$\{[0.8, \mathrm{o}, \mathrm{o}, \mathrm{0.6}, \mathrm{o}, \mathrm{o}, \mathrm{o}, \mathrm{o}]$, ['London radius 5okm', , , 'left-wing', , , , ]\}

would indicate an individual or community with a strong geographical bias towards the London area and people interested in left wing politics, with nothing else mattering. Typically a community profile might concentrate on a limited number of facets whereas that of an individual would be complete.

We want to examine the compatibility between two entities, individuals or communities; one of which will be the subject, the one looking to find a possible match and the other the object. The first stage is to examine the textual parts of the two profiles. This can be done subjectively by the subject, or by some form of artificial intelligence. The result of this analysis is to produce a match profile - that is we assign a number to represent how the textual descriptions are judged to match together - along with a brief description of how that result is achieved. This gives us now three profiles for the source, the object and the match itself. A fourth profile may then be used, a threshold, which contains minimum values which must be reached for the match on any facet to be considered viable. A simple threshold could be eight zeroes

We can now apply an algorithm to the numeric parts of those four profiles, ${ }^{21}$ to end up with an eight element vector that represents the distance between source and object. Note that this resultant vector should not be normalised since its magnitude matters. It is this vector that we can now use as the edge weight on our connection graph and use in any determination of clusters or other analysis.

Figure 2 shows some of the relationship between members of a community, and how a potential recruit (Harriet) who already follows one existing member (Colin) matches her profile against that of the 


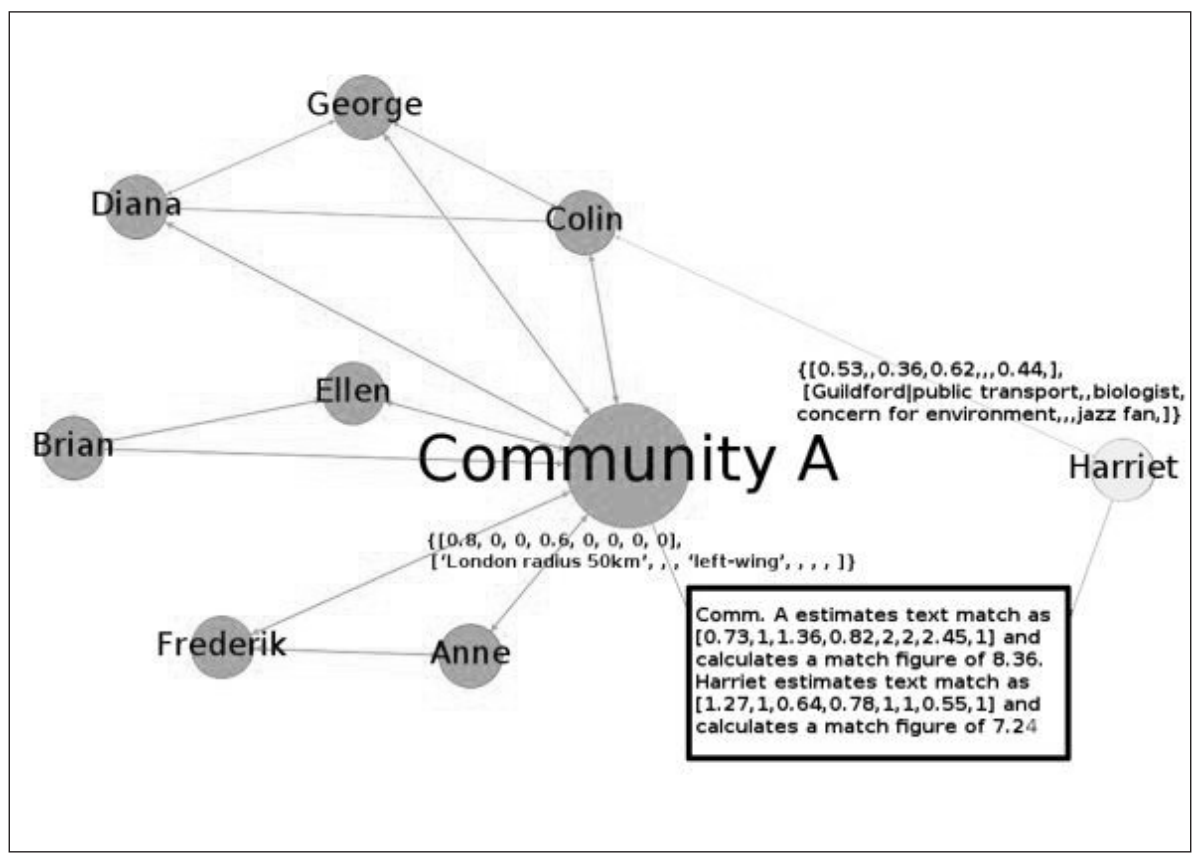

Figure 2: Relationships within a community and a potential new member Source: Author's elaboration

community. The text matches can be done subjectively or by using some textual analysis and matching tool. The algorithm used for Table 3 is a very simple one based on multiplying the difference between the profiles by the assigned text match.

If $\mathbf{f}_{\mathrm{a}}$ is a facet value for $\mathrm{A}$, and $\mathbf{f}_{\mathbf{h}}$ for $\mathrm{H}, \mathbf{t}_{\mathrm{a}}$ the text match from A's point of view and $\mathbf{t}_{\mathbf{h}}$ for $\mathrm{H}, \mathbf{m}_{\mathrm{a}}$ the value for the match from A's point of view and $\mathbf{m}_{h}$ for $\mathrm{H}$ then this simple algorithm uses the formulae:

$$
\begin{aligned}
& \mathbf{m}_{\mathbf{a}}=\left(1-\left(\mathbf{f}_{\mathbf{a}}-\mathbf{f}_{\mathbf{h}}\right)\right) * \mathbf{t}_{\mathbf{a}} \\
& \mathbf{m}_{\mathbf{h}}=\left(1-\left(\mathbf{f}_{\mathbf{h}}-\mathbf{f}_{\mathbf{a}}\right)\right) * \mathbf{t}_{\mathbf{h}}
\end{aligned}
$$

All the match values for one person can be summed to give a single figure, if required.

This does bring out how the view of the potential match differs from each side, and how one side can potentially accept the match as being over their threshold, while the other rejects it because it does not meet theirs.

We should remember that drift often occurs; individuals and communities change over time so that it is important that this analysis is repeated at regular intervals. There will also be bias in the way that any algorithm is applied. There will be individual and cultural bias in the way that the data is provided and interpreted and bias built into the algorithm itself. This is unavoidable. If the data and the algorithm are open and modifiable then such bias can be recognised so that with repetition the bias can be minimised as well as accounting for change.

\section{Conclusions}

The purpose of this article has been to show that it is possible to devise a standard, open and useful method for describing online relationships, individuals and communities. It can assist in the sometimes daunting task of navigating through the bewildering array of possibilities opened up by the Internet. The mechanism used can be both understandable and useful for people and communities and supply sufficient data for analysis of the new social structures. Modern techniques for textual analysis of descriptions of the requirements for individuals and communities can be combined with numerical algorithms to provide clear numerical values for suitability, while retaining the opportunity for human oversight and input. Graph theory and the study of mul- 
Table 1: Calculating matches Source: Author's elaboration

\begin{tabular}{|c|c|c|c|c|c|c|c|}
\hline A's profile & A text & H profile & H text & $\begin{array}{l}\text { A's text } \\
\text { match }\end{array}$ & $\begin{array}{l}\text { H's text } \\
\text { match }\end{array}$ & A's match & H's match \\
\hline 0.80 & $\begin{array}{l}\text { London ra- } \\
\text { dius } 50 \mathrm{~km}\end{array}$ & 0.53 & $\begin{array}{l}\text { Guildford public } \\
\text { transport }\end{array}$ & 1.00 & 0.60 & 0.73 & 1.27 \\
\hline 0.00 & & 0.00 & & 1.00 & 1.00 & 1.00 & 1.00 \\
\hline 0.00 & & 0.36 & biologist & 1.00 & 0.50 & 1.36 & 0.64 \\
\hline 0.60 & left-wing & 0.62 & $\begin{array}{l}\text { concern for en- } \\
\text { vironment }\end{array}$ & 0.80 & 0.60 & 0.82 & 0.78 \\
\hline 0.00 & & 0.00 & & 1.00 & 1.00 & 1.00 & 1.00 \\
\hline 0.00 & & 0.00 & & 1.00 & 1.00 & 1.00 & 1.00 \\
\hline 0.00 & & 0.45 & jazz fan & 1.00 & 0.40 & 1.45 & 0.55 \\
\hline 0.00 & & 0.00 & & 1.00 & 1.00 & 1.00 & 1.00 \\
\hline & & & & & & 8.36 & 7.24 \\
\hline
\end{tabular}

ti-dimensional spaces can be used to give insights into how online communities grow and to provide assistance in their formation.

The techniques described here are not meant to be prescriptive but to suggest possible ways in which the use of artificial intelligence and associated algorithms can be removed, or at least borrowed, from the opaque, commercial world of recommendation systems where they are regarded, often rightly, with some mistrust, into a more open and useful role. Further work will include refinement of the algorithm used to calculate numerical values to assign to a prospective match, and development of a user experience that allows both the user's subjective feelings and an AI to work together in a mutually beneficial manner to achieve the desired result. 